\title{
Prevalence of Rhinovirus and Respiratory Syncytial Virus Among Patients With Chronic Rhinosinusitis
}

\author{
Hassan Abshirini ${ }^{1} ;$ Manochher Makvandi ${ }^{2}$; Meghdad Seyyed Ashrafi ${ }^{1} ;$ Mojtaba Hamidifard $^{2}$; \\ Nader Saki ${ }^{1,}$ \\ ${ }^{1}$ Hearing and Speech Research Center, Ahvaz Jundishapur University of Medical Sciences, Ahvaz, IR Iran \\ ${ }^{2}$ Infectious and Tropical Diseases Research Centre, Ahvaz Jundishapur University of Medical Sciences, Ahvaz, IR Iran \\ *Corresponding author: Nader Saki, Hearing and Speech Research Center, Ahvaz Jundishapur University of Medical Sciences, Ahvaz, IR Iran. Fax: +98-6132921838, E-mail: saki-n@ \\ ajums.ac.irAhvaz.ent@gmail.com
}

Received: May 7, 2014; Revised: October 14, 2014; Accepted: December 30, 2014

\begin{abstract}
Background: Chronic rhinosinusitis (CRS) is one of the most common chronic illnesses, but the etiology and pathogenesis of CRS are not well understood. Few studies have been carried out on the role of viruses in patients with chronic sinusitis so far.

Objectives: Regarding the high number of patients, we intended to evaluate the prevalence of rhinovirus and respiratory syncytial virus in patients with CRS. Doing so, we may pave the way for definitely achieving the causes and factors of the disease and consequently definite treatment of this debilitating disease in future studies.

Patients and Methods: This cross-sectional study was carried out on 76 patients. Sample of the study consisted of patients with CRS who were candidates for functional endoscopic sinus surgery (FESS). The specimens were collected during FESS between February 2013 and December 2013. For this purpose, after entering into sinuses, the specimens were collected from their mucus. They were then placed in Dulbecco's modified Eagle's viral transport medium (DMEM). They were transferred to the virology lab of the university in a cold chain. The specimens were maintained in $-70^{\circ} \mathrm{C}$ before examination. Reverse transcriptase-polymerase chain reaction (RT-PCR) was applied to explore the presence of rhinovirus and respiratory syncytial virus.

Results: In this study, 76 patients with rhinosinusitis underwent FESS as qualified candidates. The sample of the study consisted of 48 males (63.2\%) and 28 females (36.8\%) with the mean age of 44.3 years and an age range of 19-76 years. Among the 76 patients, 53 were with polyps and 23 without polyps. Among the patients, 66 were candidates of FESS for the first time. Other 11 patients had previously undergone the surgery. The results from PCR indicated that 22 (28.94\%) patients had rhinovirus and 9 (11.84\%) had respiratory syncytial virus (RSV). A total of 25 patients (32.89\%) had one of the two viruses. In 6 (7.89\%) specimens, both viruses were reported.

Conclusions: CRS is a common disease with negative effects on the quality of patients' lives. This study showed the high prevalence of these two common respiratory viruses in patients with CRS.
\end{abstract}

Keywords: Sinusitis; Prevalence; Rhinovirus; Respiratory Syncytial Viruses

\section{Background}

Rhinosinusitis is the inflammation of mucus. This infection is categorized into three groups: acute $(<$ three weeks), sub-acute (4-12 weeks), and chronic ( $>12$ weeks). Chronic rhinosinusitis (CRS) is one of the most prevalent chronic diseases. About $15 \%$ of the human population has this disease. This has adverse effect on an individual's health (1). Unlike acute sinusitis where viral and bacterial factors are known as the main causes, its causes and factors are poorly recognized (2). Prospective studies are kept on to detect inflammatory mediators in CRS. Despite great advances in this field, the final cause of the up-regulation of lymphocytic and eosinophilic activities occurring in CRS is not determined. They finally result in inflammations in the mucus (3). In recent studies, there have been assumptions indicating the role of pathogenic factors, especially fungus and bacterium. Yet, few studies have been performed on the role of viruses, although their roles as an initiator in the inflammatory processes of mucus and sinus are established.

Two key factors in CRS development include complex osteomeatal obstruction (where maxillary, frontal and anterior ethmoid secretions discharge in nasal cavity) and increase of epithelial and mucociliary cells malfunction. Normally, patients with CRS report their symptoms after a viral infection. This can obstruct nasal complex osteomeatal due to inflammation. Besides, the production of inflammatory mediators and destruction of nasal epithelial cells are among other consequences of the virus (4). Rhinovirus can result in stable changes in the amount of topical cytokine and the enhancement of bacteria adhesion to mucus cells. This virus can lead to further durability of transient infections by these two mechanisms (5). Another critical issue is that the accompaniment between asthma and CRS, especially simultaneous occur-

Copyright (C) 2015, Ahvaz Jundishapur University of Medical Sciences. This is an open-access article distributed under the terms of the Creative Commons Attribution-NonCommercial 4.0 International License (http://creativecommons.org/licenses/by-nc/4.0/) which permits copy and redistribute the material just in noncommercial usages, provided the original work is properly cited. 
Abshirini $\mathrm{H}$ et al.

rence of the two diseases and nasal polyps are proved. The overlap of these two diseases causes is also demonstrated (6). Children's infection by RSV increases the asthma occurrence which is a chronic disease in the first decade. This may be due to permanent changes of mucus before removing the virus or due to the persistence of a part of virus in mucus. The fact that respiratory viruses are capable of inducing relapse and enduring the infection in human is apparently clear (7).

Regarding the abovementioned, the assumption of the viruses roles in inducing CRS is discussed in three axes: first, viruses act as the initiators of inflammation. They can also affect permanent stimulation of inflammation. Finally, these viruses can be the factors of acute exacerbated symptoms in CRS patients. Hence, regarding the above axes as well as the asthma pathogenesis overlap as a chronic disease, CRS and the approved role of respiratory viruses in inducing asthma, we decided to study the prevalence of these viruses in patients with CRS. We intended to pave the way for definite achievement to CRS causes and factors and consequently to the definite treatment of this debilitating disease in future researches. This study is important mostly because it is based on the fact that few studies have been conducted on the existence of viruses in patients with chronic sinusitis so far. Besides, the number of specimens has also been small. Therefore, the absence of virus in these studies has not been satisfying. As a result, further studies are required with larger sample sizes. Regarding the high number of patients with these viruses in this educational center, the probability of gaining positive specimens is increased. Besides, it can enhance the validity of this study for other researchers. It can also explain the cost-effectiveness of expensive case-control studies on the relationship between these viruses and the debilitating chronic sinusitis disease.

\section{Objectives}

Hence, regarding the high number of patients with this disease, we intended to evaluate the prevalence of rhinovirus and respiratory syncytial virus in patients with CRS.

\section{Patients and Methods}

This cross-sectional study was conducted on 76 patients. Sample of the study consisted of CRS candidates of functional endoscopic sinus surgery (FESS). Individuals with immunosuppressive diseases including diabetic patients were excluded from the study. Similarly, individuals who manifested common cold symptoms two week before and (or) after the admittance and (or) a week after surgery (regarding the incubation period of common cold) were excluded. Specimens were collected during FESS between February 2012 and December 2012. For that purpose, after entering into sinuses, the specimens were collected from their mucus. They were then placed in Dulbecco's modified Eagle's viral transport medium (DMEM). They were transferred to the virology lab of the Ahvaz Jundishapur University of Medical Sciences (Ahvaz, IR Iran) while keeping the cold chain. The specimens were maintained in $-70^{\circ} \mathrm{C}$ before examination. Reverse transcriptase-polymerase chain reaction (RT-PCR) was used to explore the presence of rhinovirus and respiratory syncytial virus. To do so, first, the viral genome must be extracted from the specimens.

The extraction was performed using high-pure viral nucleic acid kit (Roche, Germany). Since these were RNA viruses, first-strand cDNA synthesis kit (Thermo Scientific, USA) was used to synthesize cDNA from viral RNA. About $5 \mu \mathrm{L}$ of the cDNA synthesis product was used for PCR. The final volume was adjusted to $50 \mu \mathrm{L}$ for the test. PCR included the following compounds: $5 \mu \mathrm{L}$ cDNA synthesis product, $1 \mu \mathrm{L}$ dNTP $10 \mathrm{mM}, 0.3 \mu \mathrm{LTaq}$ polymerase enzyme, $5 \mu \mathrm{L}$ PCR buffer 10x, $1 \mu \mathrm{L}$ of each primer (Table 1 ) with $30 \mathrm{PM}$ and $36.7 \mu \mathrm{L}$ DNase-free water. All these substances were prepared from Arian Gen Gostar Company (Iran). Thermocycler was organized to perform PCR stages as follows: five minutes of initial denaturation in $95^{\circ} \mathrm{C}$, then 40 cycles including 40 seconds of denaturation in $95^{\circ} \mathrm{C}, 40$ seconds of annealing in $40^{\circ} \mathrm{C}, 45$ seconds of extension in $72^{\circ} \mathrm{C}$, and at the end, three minutes of final extension in $72^{\circ} \mathrm{C}$. After PCR, $10 \mu \mathrm{L}$ of the PCR product was analyzed by electrophoresis in $1.5 \%$ agarose gel to check the existence of PCR band (8).

\begin{tabular}{lr}
\hline \multicolumn{2}{l}{ Table 1. Primers Sequences } \\
\hline Primer & $\mathbf{5}^{\prime} \rightarrow \mathbf{3}^{\prime}$ Sequence \\
\hline RV F & GCA CTT CTG TTT CCC C \\
RV R & GGC AGC CAC GCA GGC T \\
RSV F & AAT ACA GCM AAA TCT AAC CAA CTT TAC A \\
RSV R & GCC AAG GAA GCA TGC AAT AAA \\
\hline
\end{tabular}

\section{Results}

In this study, 76 patients with rhinosinusitis underwent surgery as qualified candidates. The sample of the study consisted of 48 males (63.2\%) and 28 females (36.8\%) with the mean age of 44.3 and an age range of 19-76 years. Among the 76 patients, 53 were with polyps and 23 without polyps. Again, 66 patients were candidates of FESS for the first time. The other 11 patients previously ender went surgery. The results from RT-PCR indicated that 22 (28.94\%) patients had rhinovirus and $9(11.84 \%)$ had RSV. A total of 25 patients (32.89\%) had one of the two viruses. In $6(7.89 \%)$ specimens, both viruses were reported.

\section{Discussion}

CRS is one of the most common chronic diseases. About $15 \%$ of the human population has this disease. This has adverse effects on an individual's health (1). Unlike acute sinusitis where viral and bacterial factors are known as the main causes, its causes and factors are barely recognized. 
A variety of inflammatory factors with different degrees can play a role in inducing this disease. A number of respiratory viruses can lead to acute sinusitis symptoms. It can also leave long-term effects on respiratory mucus $(3,9)$. Viral diseases can induce relapse and endure infection in human's tissues (10). A few studies have been performed using PCR to show the existence of respiratory viruses in CRS patients' specimens. In Korea, Cho et al. (11) examined specimens from nasal lavage as well as from middle meatus mucus among 111 patients with CRS. They reported one or two respiratory viruses in 56 (50\%) patients in specimens from lavage and 71 (64\%) patients in specimens from nasal mucus. The most prevalent virus (12\%) was rhinovirus, extracted from lavage specimens. It had a considerable difference with other viruses in mucus specimens from 35 (31.4\%) patients (11). In a study by Ramadan et al. (8) $20 \%$ of patients with CRS had RSV in their mucus. Yet, adenovirus was observed in none of them. Jang et al. carried out a similar study; they discovered RSV in epithelial cells specimens of $21 \%$ of patients with CRS, but nothing was observed in control group (12). The fact that the respiratory viruses are capable of inducing relapse and enduring infection in human is clear. In recent studies, their role in inducing inflammation has been shown. In case-control case studies performed before, generally, there were significant differences between case and control groups regarding the outbreak of respiratory diseases among chronic sinusitis; for instance, Cho et al. (11) and Anselmo Lima et al. (13). Accordingly, studies on the basis of liable discovery of this virus to find out special causes of the disease as well as a more effective and specific treatment for this disease are reasonable.

In this study, the prevalence of rhinovirus and respiratory syncytial virus, which are among respiratory viruses, were studied in CRS candidates of FESS. In our study, the prevalence of at least one of respiratory viruses was $32.89 \%$. Yet, it was about $64 \%$ in Cho et al. study (11). This difference is probably due to the matter that Cho et al. analyzed all respiratory viruses. Furthermore, in our study, the prevalence of specimens with two viruses was $7.89 \%$, as compared with $12.41 \%$ in Cho et al. study (11). In this study, the prevalence of rhinovirus was reported $28.94 \%$ which had a significant difference with the RSV prevalence $(11.84 \%)$. In other studies on the prevalence of rhinovirus, there were no significant differences between their results and our study results. For example, Jang et al. in South Korea reported the rhinovirus prevalence as $21 \%$ (12). In a study, Anselmo Lima et al. reported rhinovirus prevalence $22.85 \%$ (13). Moreover, this was in accordance with other studies including the one from Cho et al. who identified rhinovirus outbreak as the most prevalent virus (compared to other respiratory viruses) (11).

The strength of our study was that we collected the specimens from sinus mucus (compared to Cho et al. study who collected specimens from lower turbinate). The results support the relationship between rhinovirus and chronic sinusitis further. However, considering RSV as one of the prevalent respiratory viruses, its relationship with asthma (as a respiratory mucusal disease) has been approved in previous studies. Nevertheless, there are few studies in which it is determined as the specific cause of chronic rhinosinusitis. Its prevalence was $11.84 \%$ in our study compared with $10.8 \%$ in Cho et al. study (11). In addition, the prevalence was reported $20 \%$ in Ramadan et al. study (8). In another study by Agnieszka et al. in which IgG against all respiratory viruses was measured in CRS patients with polyps, IgG against RSV was reported in 95\% of patients (14). With respect to various statistics of this virus prevalence, further studies with concentration on this virus on the basis of case-control studies are reasonable. As a result, it will be possible to achieve significant statistics regarding the prevalence of this virus among patients with CRS similar to what is gained on rhinovirus.

Chronic rhinosinusitis is a common disease with negative effects on the quality of patients' lives. Yet, unlike acute rhinosinusitis, its causes are poorly known. Viruses (especially respiratory viruses) were among the inflammatory causes examined in previous studies and measured in terms of prevalence. Their prevalence was significant in patients compared to control groups. Rhinovirus was the most prevalent respiratory virus. In our study, the prevalence of this virus was almost same as the previous investigations. The relationship between RSV and chronic respiratory asthma was emphasized in previous studies. The results of this study were slightly different from other studies on patients with chronic rhinosinusitis. More accurate and expensive case-control studies on this virus are needed to achieve significant relationship between this virus and chronic rhinosinusitis and to find out a more effective and specific treatment for this disease.

\section{Acknowledgements}

We would like to appreciate Meghdad Seyyed Ashrafi an ear, nose and throat specialist, by the contribution of whom this study could be funded.

\section{Authors' Contributions}

All investigators participated in trial design, conduct, analysis, and the writing of the paper, and took part in regular meetings before, during, and after completion of the trial.

\section{Funding/Support}

Ahvaz Jundishapur University of Medical Sciences, Ahvaz, IR Iran funded this study.

\section{References}

1. Benninger MS, Ferguson BJ, Hadley JA, Hamilos DL, Jacobs M, Kennedy DW, et al. Adult chronic rhinosinusitis: definitions, diagnosis, epidemiology, and pathophysiology. Otolaryngol Head Neck Surg. 2003;129(3 Suppl):S1-32.

2. Ahn BH, Park YH, Shin SH. Mouse model of Aspergillus and Alter- 
naria induced rhinosinusitis. Auris Nasus Larynx. 2009;36(4):4226.

3. Healy DY, Leid JG, Sanderson AR, Hunsaker DH. Biofilms with fungi in chronic rhinosinusitis. Otolaryngol Head Neck Surg. 2008;138(5):641-7.

4. Clement S, Vaudaux P, Francois P, Schrenzel J, Huggler E, Kampf S, et al. Evidence of an intracellular reservoir in the nasal mucosa of patients with recurrent Staphylococcus aureus rhinosinusitis. J Infect Dis. 2005;192(6):1023-8.

5. Shin SH, Ponikau JU, Sherris DA, Congdon D, Frigas E, Hom burger HA, et al. Chronic rhinosinusitis: an enhanced immune response to ubiquitous airborne fungi. J Allergy Clin Immunol. 2004;114(6):1369-75.

6. Bachert C, Zhang N, Patou J, van Zele T, Gevaert P. Role of staphylococcal superantigens in upper airway disease. Curr Opin Allergy Clin Immunol. 2008;8(1):34-8.

7. Ha KR, Psaltis AJ, Tan L, Wormald PJ. A sheep model for the study of biofilms in rhinosinusitis. Am J Rhinol. 2007;21(3):339-45.

8. Ramadan HH, Farr RW, Wetmore SJ. Adenovirus and respiratory syncytial virus in chronic sinusitis using polymerase chain reaction. Laryngoscope. 1997;107(7):923-5.
9. Wang J, Matsukura S, Watanabe S, Adachi M, Suzaki H. Involvement of Toll-like receptors in the immune response of nasal polyp epithelial cells. Clin Immunol. 2007;124(3):345-52.

10. Neumann R, Genersch E, Eggers HJ. Detection of adenovirus nucleic acid sequences in human tonsils in the absence of infectious virus. Virus Res. 1987;7(1):93-7.

11. Cho GS, Moon BJ, Lee BJ, Gong CH, Kim NH, Kim YS, et al. High rates of detection of respiratory viruses in the nasal washes and mucosae of patients with chronic rhinosinusitis. J Clin Microbiol. 2013;51(3):979-84.

12. Jang YJ, Kwon HJ, Park HW, Lee BJ. Detection of rhinovirus in turbinate epithelial cells of chronic sinusitis. Am J Rhinol. 2006;20(6):634-6.

13. Anselmo Lima WT, Paula FE, Lima J, Modena JLP, Arruda E, Valera $\mathrm{F}$, et al. Prevalence of Respiratory Viruses in Chronic Rhinosinusitis and Its Influence on the Mucosa. Otolaryngol Head Neck Surg. 2013;149(2 Suppl):263.

14. Agnieszka OZ, Marzanna, J. , Marek L. . Kowalski.the immune response against respiratory pathogens in pathent with chronic rhinosinusitisnasal polyps and asthma with or without sensivity to aspirin. World Allergy Organ J. 2012;5(2):S64. 